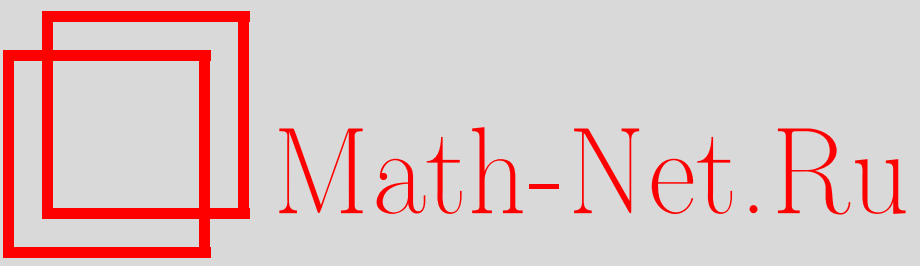

Г. В. Балакин, О решении некоторых классов систем булевых уравнений рекуррентного типа, Матем. вопр. криптогр., 2013, том 4, выпуск 1, 5-25

DOI: https://doi.org/10.4213/mvk71

Использование Общероссийского математического портала Math-Net.Ru подразумевает, что вы прочитали и согласны с пользовательским соглашением

http://www.mathnet.ru/rus/agreement

Параметры загрузки:

IP : 34.239 .49 .27

26 апреля 2023 г., 17:14:12 
УДК 519.212.2

\title{
О решении некоторых классов систем булевых уравнений рекуррентного типа
}

\author{
Балакин Г. В. \\ Академия криптографии Российской Федерации, Москва
}

Получено 20.IV.2012

В работе изучаются системы булевых уравнений рекуррентного типа. Основное внимание уделяется решению систем с рекуррентой, которая порождается трёхчленным соотношением. В частности, предложен метод восстановления искажённой рекурренты, основанный на решении псевдобулевых линейных уравнений и неравенств. Приведены конкретные примеры.

Ключевые слова: булевы уравнения, псевдобулевы линейные уравнения и неравенства, рекуррента

On the solution of some classes of Boolean equations systems of recurrent type

\section{G. V. Balakin}

Academy of Cryptography of the Russian Federation, Moscow

\begin{abstract}
Systems of Boolean equations of recurrent type are considered. We concentrate mainly on systems generated by the three-term recurrent equation. In particular, a method of noisy recurrent sequence reconstruction is proposed; this method is based on the solution of pseudo-Boolean linear equations and inequalities. Concrete examples are provided.
\end{abstract}

Key words: Boolean equations, pseudo-Boolean linear equations and inequalities, recurrent sequence

Citation: Mathematical Aspects of Cryptography, 2013, vol. 4, no. 1, pp. 5-25 (Russian).

(C) 2013 Балакин Г. В. 


\section{§ 1. Постановка задачи}

Рассмотрим систему булевых уравнений следующего вида:

$$
F\left(\bar{x}_{i}\right)=a_{i}, \quad i=1,2, \ldots, t .
$$

Здесь последовательность $x_{1}, x_{2}, \ldots$ подчиняется некоторому рекуррентному соотношению, начальный участок рекурренты $\bar{x}_{1}=\left(x_{1}, \ldots, x_{n}\right)$, a $\bar{x}_{i}=\left(x_{i}, x_{i+1}, \ldots, x_{i+n-1}\right), i=2,3, \ldots, t$.

Любая система (1) может быть представлена в ином виде:

$$
F_{1}\left(\bar{x}_{i}\right) \oplus F_{2}\left(\bar{x}_{i}\right) \cdot F_{3}\left(\bar{x}_{i}\right)=a_{i}, i=1,2, \ldots, t .
$$

Причём степени многочленов $F_{1}, F_{2}, F_{3}$ не выше степени многочлена $F$, а в ряде случаев даже существенно меньше. В работах $[1,2]$ показано, что $i$-е $(i=1,2, \ldots, t)$ уравнение из системы (2) эквивалентно псевдобулеву равенству

$$
Q_{1 i}\left(\bar{x}_{i}\right)+Q_{2 i}\left(\bar{x}_{i}\right)+Q_{3 i}\left(\bar{x}_{i}\right)+\left[Q_{1 i}\left(\bar{x}_{i}\right) \oplus Q_{2 i}\left(\bar{x}_{i}\right) \oplus Q_{3 i}\left(\bar{x}_{i}\right) \oplus 1\right]=1,
$$

где

$$
\begin{aligned}
& Q_{1 i}\left(\bar{x}_{i}\right)=F_{1}\left(\bar{x}_{i}\right) \oplus a_{i}, \\
& Q_{2 i}\left(\bar{x}_{i}\right)=F_{1}\left(\bar{x}_{i}\right) \oplus F_{2}\left(\bar{x}_{i}\right) \oplus a_{i}, \\
& Q_{3 i}\left(\bar{x}_{i}\right)=F_{1}\left(\bar{x}_{i}\right) \oplus F_{3}\left(\bar{x}_{i}\right) \oplus a_{i} .
\end{aligned}
$$

Не исключено, что внутри одной из трёх квадратных скобок будет находиться линейная функция. Очевидно, что уравнение (3) равносильно следующему псевдобулеву неравенству:

$$
Q_{1 i}\left(\bar{x}_{i}\right)+Q_{2 i}\left(\bar{x}_{i}\right)+Q_{3 i}\left(\bar{x}_{i}\right) \leq 1 .
$$

Неравенство (4) можно представить в виде пары условий

$$
Q_{1 i}\left(\bar{x}_{i}\right)+Q_{2 i}\left(\bar{x}_{i}\right)+Q_{3 i}\left(\bar{x}_{i}\right)=\theta_{i}, \quad \theta_{i} \in\{0,1\},
$$

равносильной неравенству (4) относительно решений $\bar{x}_{i}$.

Неравенство (4) характеризует (определяет) пороговую функцию от трёх аргументов, в качестве последних могут выступать линейные формы от членов рекурренты. В псевдобулевом условии (5) величина $\theta_{i}$ является мешающим параметром [1].

Теорема 1. Уравнение (2) при $a_{i}=0$ эквивалентно псевдобулеву неравенству

$$
X_{i}+Y_{i}+Z_{i} \leq 1,
$$


а при $a_{i}=1-$-ругому неравенству

$$
X_{i}+Y_{i}+Z_{i} \geq 2
$$

где

$$
X_{i}=F_{1}\left(\bar{x}_{i}\right), \quad Y_{i}=F_{1}\left(\bar{x}_{i}\right) \oplus F_{2}\left(\bar{x}_{i}\right), \quad Z_{i}=F_{1}\left(\bar{x}_{i}\right) \oplus F_{3}\left(\bar{x}_{i}\right) .
$$

Доказательство. В неравенство (4) подставим значение $a_{i}=0$, получим первое неравенство из теоремы. При $a_{i}=1$ находим, что

$$
\left[F_{1}\left(\bar{x}_{i}\right) \oplus 1\right]+\left[F_{1}\left(\bar{x}_{i}\right) \oplus F_{2}\left(\bar{x}_{i}\right) \oplus 1\right]+\left[F_{1}\left(\bar{x}_{i}\right) \oplus F_{3}\left(\bar{x}_{i}\right) \oplus 1\right] \leq 1
$$

или в другой записи

$$
3-X_{i}-Y_{i}-Z_{i} \leq 1 \text {. }
$$

Это доказывает справедливость второго неравенства. Теорема доказана.

По существу теорема 1 утверждает, что функция $F\left(\bar{x}_{i}\right)$ из системы (1) заменяется пороговой функцией $\Phi\left(X_{i}, Y_{i}, Z_{i}\right)$ относительно трёх неизвестных $X_{i}, Y_{i}, Z_{i}$. Мы будем рассматривать случай, когда среди функций $F_{1}, F_{2}, F_{3}$ есть линейные, а исходная рекуррента является трёхчленной

$$
x_{i} \oplus x_{i+k} \oplus x_{i+n}=0,
$$

$k$ и $n-$ взаимно простые числа. Требуется, используя представления (2), (3), (4), (5) и теорему 1, найти рекуррентную последовательность, удовлетворяющую системе (1).

Во втором пункте приводятся метод решения поставленной задачи в общем случае и некоторые конкретные расчёты для случая, когда функция $F$ существенно зависит ровно от $r \geq 3$ переменных.

Далее в $\S 3$ рассматривается случай, когда функция $F$ из системы (1) является пороговой от трёх аргументов - линейных функций. Приведён метод решения, использующий псевдобулевы равенства и неравенства для линейных булевых функций.

В $\ 4$ рассматривается задача восстановления искажённой трёхчленной рекурренты. Метод решения основан на исследовании ограничений на ошибки. Приведён простейший пример, демонстрирующий различные приёмы определения значений ошибок и членов рекурренты.

Для четырёхчленных рекуррент в $§ 5$ предложен и продемонстрирован на примере метод решения задачи нахождения исходной рекурренты.

В заключение в $\S 6$ приводится один из возможных критериев различения случайной системы уравнений и заведомо совместной системы уравнений. 
Для случайной системы уравнений (ССУ, см. [3]) полагаем, что последовательность правых частей системы (1) состоит из реализаций независимых равновероятно распределённых случайных величин. Если перед нами заведомо совместная случайная система уравнений (ЗСССУ, см. [3]), то считаем при расчётах, что любая комбинация из небольшого числа $s<n$ значений алгебраически независимых линейных форм имеет вероятность появления, равную $(1 / 2)^{s}$. Это предположение может быть оправдано, если начальное заполнение рекуррент и три ненулевые линейные формы выбираются случайно и равновероятно из соответствующих множеств возможных значений.

\section{§ 2. Общий случай}

В общем случае исходную функцию $F$ из системы (1) можно заменить линейным булевым статистическим аналогом и решать задачу восстановления искажённой рекуррентной последовательности. Если же функция $F$ является пороговой, то задача сводится к решению системы линейных псевдобулевых неравенств. Некоторые методы решения таких систем приведены в работах $[4,5]$. Но в обоих случаях эти методы решения задачи в силу разных причин могут оказаться неэффективными. С другой стороны, существуют эффективные методы решения задачи для частных случаев. Ниже будут изложены некоторые из них.

Если функция $F_{1}\left(\bar{x}_{i}\right)=L_{i}\left(\bar{x}_{i}\right)$, где $L_{i}\left(\bar{x}_{i}\right)$ является линейной однородной функцией и при случайном и равновероятном выборе вектора $\bar{x}_{1}=\left(x_{1}, \ldots, x_{n}\right)$ вероятность события $\left\{F_{2}\left(\bar{x}_{i}\right) F_{3}\left(\bar{x}_{i}\right)=1\right\}$ равна $1 / 4$, то систему (2) можно рассматривать как линейную систему уравнений с искажённой правой частью

$$
L_{1}\left(\bar{x}_{i}\right)=a_{i} \oplus \varepsilon_{i}, i=1,2, \ldots, t
$$

с ошибками

$$
\varepsilon_{i}=F_{2}\left(\bar{x}_{i}\right) F_{3}\left(\bar{x}_{i}\right), \quad p=\mathbf{P}\left\{\varepsilon_{i}=1\right\}=\frac{1}{4}, \quad i=1,2, \ldots, t .
$$

Если $\varepsilon_{1}=1$, то будем говорить, что произошло искажение $i$-го знака правой части рассматриваемой линейной системы уравнений.

Используя соотношение (6), находим, что

$$
\begin{gathered}
\mathcal{E}_{i} \oplus \varepsilon_{i+k} \oplus \varepsilon_{i+n}=a_{i} \oplus a_{i+k} \oplus a_{i+n}, \\
\varepsilon_{i} \oplus \varepsilon_{i+2^{s} k} \oplus \varepsilon_{i+2^{s} n}=a_{i} \oplus a_{i+2^{s} k} \oplus a_{i+2^{s} n}, s \geq 1 .
\end{gathered}
$$


Пусть число соотношений (7), содержащих $\varepsilon_{i}$, будет $r_{i}$, среди них $r_{i 1}$ с правой частью, равной 1 , и $r_{i}-r_{i 1}=r_{i 0}$ с правой частью, равной 0 . Тогда, полагая, что в этих соотношениях число $\varepsilon_{j}, j \neq i$, равно $2 r_{i}$ и все они статистически независимы, приходим к формуле

$$
\mathbf{P}\left\{\varepsilon_{i}=1 \mid r_{i 1}, r_{i 0}\right\}=\left[1+3\left(\frac{\mathbf{P}\left\{\varepsilon_{i+k} \oplus \varepsilon_{i+n}=1\right\}}{\mathbf{P}\left\{\varepsilon_{i+k} \oplus \varepsilon_{i+n}=0\right\}}\right)^{r_{i 1}-r_{i 0}}\right]^{-1}=\left[1+3\left(\frac{3}{5}\right)^{r_{i 1}-r_{i 0}}\right]^{-1} .
$$

Более подробно переоценки вероятностей приведены в работе [6].

Приведём некоторые конкретные значения вероятностей $\mathbf{P}\left\{\varepsilon_{i}=1 \mid r_{i 1}, r_{i 0}\right\}$. Из формулы (8) находим

$$
\begin{gathered}
\mathbf{P}\left\{\varepsilon_{i}=1 \mid r_{i 1}-r_{i 0}=3\right\}=\left[1+3\left(\frac{3}{5}\right)^{3}\right]^{-1} \approx 0,607, \\
\mathbf{P}\left\{\varepsilon_{i}=1 \mid r_{i 1}-r_{i 0}=-3\right\} \approx 0,07 \\
\mathbf{P}\left\{\varepsilon_{i}=1 \mid r_{i 1}-r_{i 0}=5\right\}=\left[1+3\left(\frac{3}{5}\right)^{5}\right]^{-1} \approx 0,81 \\
\mathbf{P}\left\{\varepsilon_{i}=1 \mid r_{i 1}-r_{i 0}=-5\right\} \approx 0,025
\end{gathered}
$$

Самые надёжные значения некоторых ошибок подставляем в уравнения (7) и получаем двучленные уравнения.

Иногда целесообразно находить совместные апостериорные распределения для двух, трёх и так далее ошибок. Например, пусть $p=1 / 4$, известно, что $\varepsilon_{i} \oplus \varepsilon_{i+k}=0$, и известны значения величин $r_{i 1}, r_{i 0}, r_{i+k, 1}, r_{i+k, 0}$. Все ошибки разные и статистически независимые. Тогда

$$
\mathbf{P}\left\{\varepsilon_{i}=\varepsilon_{i+k}=0 \mid \varepsilon_{i} \oplus \varepsilon_{i+k}=0, r_{i 1}, r_{i 0}, r_{i+k, 1}, r_{i+k, 0}\right\}=\left[1+\frac{1}{9}\left(\frac{5}{3}\right)^{r_{i 1}-r_{i 0}+r_{i+k, 1}-r_{i+k, 0}}\right]^{-1} .
$$

Если $x_{i_{1}}=x_{i_{2}}=\ldots=x_{i_{s}}, a_{i_{1}}=a_{i_{2}}=\ldots=a_{i_{s}}$, то

$$
\begin{gathered}
\mathbf{P}\left\{x_{i_{m}}=a_{i_{1}}, m=1, \ldots, s \mid a_{i_{1}}=a_{i_{2}}=\ldots=a_{i_{s}}\right\}= \\
=\left(\frac{3}{4}\right)^{s}\left[\left(\frac{3}{4}\right)^{s}+\left(\frac{1}{4}\right)^{s}\right]^{-1}=\left[1+\left(\frac{1}{3}\right)^{s}\right]^{-1} .
\end{gathered}
$$

2013, T. 4, № 1, C. 5-25 
Другой пример. Пусть в каждом уравнении системы (1) присутствуют $r \geq 3$ неизвестных членов рекуррентной последовательности, порождённой исходным трёхчленным соотношением (6). Совокупность $r s$ неизвестных, входящих в уравнения с номерами $i, i+k, \ldots, i+k(s-1), s \leq n$, может принимать $2^{r s}$ значений. Отберём примерно $2^{s(r-1)}$ вариантов значений этих неизвестных, удовлетворяющих выбранным $s$ уравнениям. Эти варианты должны удовлетворять и уравнениям с номерами

$$
\begin{aligned}
& n+i, n+i+k, \ldots, n+i+(s-2) \cdot k, \\
& 2 n+i, 2 n+i+k, \ldots, 2 n+i+(s-3) \cdot k, \\
& \ldots \\
& (s-1) \cdot n+i .
\end{aligned}
$$

Число проверочных уравнений для взаимно простых чисел $n$ и $k$ равно

$$
(s-1)+(s-2)+\ldots+1=s(s-1) / 2 .
$$

Таким образом, если функция $F$ случайная и равновероятная, то среднее число вариантов $r s$ неизвестных, удовлетворяющих $s(s+1) / 2$ уравнениям, равно

$$
N(s)=2^{r s-s(s+1) / 2}
$$

Величина $N(s)$ с ростом $s$ вначале возрастает, а затем начинает убывать с $s=r$ и, наконец, при $s=2 r-1$ величина $N(s)$ становится не больше единицы. Трудозатраты этой процедуры отбора допустимых вариантов пропорциональны величине

$$
\begin{gathered}
T(r)=N(1)+N(2)+\ldots+N(2 r-1)= \\
=2^{r-1}+2^{2 r-3}+\ldots+\frac{1}{2} 2^{r(r-1) / 2}+2^{r(r-1) / 2}+2^{r(r-1) / 2}+\frac{1}{2} 2^{r(r-1) / 2}+\frac{1}{8} 2^{r(r-1) / 2}+\ldots+1 .
\end{gathered}
$$

Для больших значений $r$ величина $T(r)<3,3 \cdot 2^{r(r-1) / 2}$, а для $r=5$ точное значение $T(5)$ равно

$$
2^{4}+2^{7}+2^{9}+2^{10}+2^{10}+2^{9}+2^{7}+2^{4}+2^{0}=2^{10} \cdot 3,28 .
$$

Если в исходных $s=2 r-1$ уравнениях нет общих неизвестных и все $r s$ неизвестных членов рекурренты независимы, то процедуру придётся повто- 
рить примерно $[n / r(2 r-1)]$ раз. Трудозатраты такого метода определения всех членов рекурренты будут пропорциональны величине

$$
T=T(r) n / r^{2}=O\left(2^{r(r-1) / 2} n / r^{2}\right) .
$$

Недостатком этого метода является необходимость иметь не меньше $(2 r-2) n$ уравнений в исходной системе. Это число заметно сокращается, если имеются общие неизвестные, например, в уравнениях с номерами $i, i+k, i+2 k, \ldots$, так как в этом случае сокращается число рассматриваемых допустимых вариантов.

Эта же задача может решаться и несколько иным способом, идея которого изложена в [7]. Предположим, что в $i$-м уравнении все $r$ неизвестных равны нулю; вероятность такого события равна $2^{-r}$. Тогда неизвестные в $(k+i)$-м и $(n+i)$-м уравнениях на соответствующих одинаковых местах должны принять одинаковые значения. Это следует из рекуррентного соотношения

$$
x_{n+i}=x_{k+i} \oplus x_{i}, \quad i \geq 0 .
$$

Следовательно, при этом предположении должно выполняться равенство

$$
F\left(\bar{x}_{k+i}\right)=F\left(\bar{x}_{n+i}\right) .
$$

Аналогичные равенства выполняются для всех $v$ трёхчленных соотношений, в которые входит $x_{i}$. Если наше предположение не выполняется, то вероятность выполнения указанных $v$ равенств равна $(1 / 2)^{v}$.

Прежде чем переходить к идеям методов решения систем уравнений вида (2) с $F_{1}\left(\bar{x}_{i}\right)=x_{i}$, сделаем два замечания. Так как ошибки и члены рекурренты связаны простыми линейными уравнениями

$$
x_{i}=a_{i} \oplus \varepsilon_{i}, i=1,2, \ldots, t,
$$

то целесообразно находить ошибки, так как они априори распределены неравновероятно, а члены рекурренты по сделанному допущению равновероятны. Более того, из линейного уравнения

$$
L\left(x_{1}, x_{2}, \ldots, x_{t}\right)=b
$$

следует равенство для ошибок

$$
L\left(\varepsilon_{1}, \varepsilon_{2}, \ldots, \varepsilon_{t}\right)=b \oplus L\left(a_{1}, a_{2}, \ldots, a_{t}\right) .
$$

Второе замечание касается необходимого числа определившихся неизвестных. Для рекурренты достаточно найти $n$ линейно независимых членов, это же замечание относится и к ошибкам. 


\section{§ 3. Случай, когда $F-$ пороговая функция от трёх аргументов (линейных функций)} имеет вид

Рассмотрим простейший случай, когда исходная система уравнений (1)

$$
F\left(X_{i}, Y_{i}, Z_{i}\right)=a_{i}, \quad i=1,2, \ldots, t .
$$

В этой системе $X_{i}, Y_{i}, Z_{i}$ являются разными линейными функциями от $x_{i}, x_{i+1}, \ldots, x_{i+n-1}$, функция $F-$ пороговая:

$$
X_{i}+Y_{i}+Z_{i} \geq 2 \text { при } F\left(X_{i}, Y_{i}, Z_{i}\right)=1
$$

и

$$
X_{i}+Y_{i}+Z_{i} \leq 1 \text {, если } F\left(X_{i}, Y_{i}, Z_{i}\right)=0 .
$$

Линейные формы $X_{i}, Y_{i}, Z_{i}$ удовлетворяют равенству (6).

Введём обозначения: $S_{i}$ - подсистема системы (9), состоящая из трёх уравнений с номерами $i, i+k, i+n, \quad$ вектор $V_{i}=\left(a_{i}, a_{i+k}, a_{i+n}\right)$ назовём весом подсистемы $S_{i}, i=1,2, \ldots, t-n$.

Лемма 1. Все 6 ремений подсистемь $S_{i}$ веса $V_{i}=(1,1,1)$ относительно шести неизвестных $X_{i}, Y_{i}, Z_{i}, X_{i+k}, Y_{i+k}, Z_{i+k}$ и только они являются решениями системы из трёх псевдобулевых равенств

$$
\begin{gathered}
X_{i}+Y_{i}+Z_{i}=2, \\
X_{i+k}+Y_{i+k}+Z_{i+k}=2, \\
\left(X_{i} \oplus X_{i+k}\right)+\left(Y_{i} \oplus Y_{i+k}\right)+\left(Z_{i} \oplus Z_{i+k}\right)=2 .
\end{gathered}
$$

Доказательство. Вариант $X_{i}=Y_{i}=Z_{i}=1$ не удовлетворяет подсистеме $S_{i}$ с весом $(1,1,1)$, поэтому верны два первых равенства из (10). Третье равенство из (10) можно записать в ином виде

$$
X_{i} X_{i+k}+Y_{i} Y_{i+k}+Z_{i} Z_{i+k}=1 .
$$

Поэтому число решений системы (10) равно 6 и все они удовлетворяют подсистеме $S_{i}$.

Лемма 2. Все 6 решений подсистемь $S_{i}$ веса $V_{i}=(1,0,0)$ относительно шести неизвестных $X_{i}, Y_{i}, Z_{i}, X_{i+k}, Y_{i+k}, Z_{i+k}$ и только они являются решениями системы из трёх псевдобулевых равенств

$$
X_{i}+Y_{i}+Z_{i}=2,
$$




$$
\begin{gathered}
X_{i+k}+Y_{i+k}+Z_{i+k}=1, \\
\left(X_{i} \oplus X_{i+k}\right)+\left(Y_{i} \oplus Y_{i+k}\right)+\left(Z_{i} \oplus Z_{i+k}\right)=1 .
\end{gathered}
$$

Доказательство аналогично доказательству леммы 1 .

Замечание 1. Лемма 2 верна и для двух других весов $(0,1,0)$ и $(0,0,1)$ с соответствующей перестановкой правых частей в (11). По существу это следует из симметрии.

Замечание 2. Уместно заметить, что при необходимости первые два линейных псевдобулевых уравнения в системах (10) и (11) можно заменить булевыми линейными уравнениями. Булев вариант лемм 1 и 2 был сообщён автору Ф. М. Малышевым. Универсальный метод получения линейных булевых следствий предложен автором в работе [1, теорема 2].

Остальные четыре подсистемы $S_{i}$ с весами, отличными от рассмотренных в леммах 1,2 , имеют по 10 решений. В частности, решения подсистемы $S_{i}$ веса $(0,0,0)$ удовлетворяют псевдобулевым неравенствам

$$
\begin{aligned}
& X_{i}+Y_{i}+Z_{i} \leq 1, \quad X_{i+k}+Y_{i+k}+Z_{i+k} \leq 1, \\
& X_{i}+Y_{i}+Z_{i+k} \leq 1, \quad X_{i}+Y_{i+k}+Z_{i+k} \leq 1, \\
& Y_{i}+Z_{i}+X_{i+k} \leq 1, \quad Z_{i}+X_{i+k}+Y_{i+k} \leq 1 .
\end{aligned}
$$

Из подсистем уравнений $S_{i}$ с весами $(1,1,0),(1,0,1),(0,1,1)$ достаточно рассмотреть одну, например с весом $(1,1,0)$. Решения такой подсистемы удовлетворяют псевдобулевым неравенствам

$$
\begin{aligned}
& X_{i}+Y_{i}+Z_{i} \geq 2, \quad X_{i+k}+Y_{i+k}+Z_{i+k} \geq 2, \\
& X_{i}+Y_{i}+Z_{i+k} \geq 2, \quad X_{i}+Y_{i+k}+Z_{i+k} \geq 2, \\
& Y_{i}+Z_{i}+X_{i+k} \geq 2, \quad Z_{i}+X_{i+k}+Y_{i+k} \geq 2 .
\end{aligned}
$$

Заметим, что левые части этих неравенств совпадают с левыми частями неравенств для подсистем $S_{i}$ веса $(0,0,0)$.

Последовательности $\bar{X}, \bar{Y}, \bar{Z}$ могут быть переставлены $3 !=6$ способами. Мы можем избавиться от этой эквивалентности, если зафиксируем значения некоторых знаков этих последовательностей. В частности, в приведённом выше первом примере (лемма 1) можно положить $X_{i}=0, Y_{i}=1, Z_{i}=1$. Тогда остальные два равенства

$$
\begin{gathered}
X_{i+k}+Y_{i+k}+Z_{i+k}=2, \\
X_{i+k}-Y_{i+k}-Z_{i+k}=0
\end{gathered}
$$


выполняются только при $X_{i+k}=1, Y_{i+k}+Z_{i+k}=1$. Здесь можно дополнительно положить $Y_{i+k}=0, Z_{i+k}=1$, и мы находим единственное решение относительно шести неизвестных $X_{i}, Y_{i}, Z_{i}, X_{i+k}, Y_{i+k}, Z_{i+k}$. Из этого решения можно получить остальные решения. Вектор, составленный из значений этих шести неизвестных, можно назвать представителем множества решений.

Далее для $t=2 n$ мы будем находить такие подсистемы уравнений из системы (9), которые состоят из трёх подсистем $S_{i}, S_{i+k}, S_{i+2 k}$ и имеют единственное решение относительно трёх неизвестных $\left(X_{i+n+k}, Y_{i+n+k}, Z_{i+n+k}\right)$.

Обозначим через $A_{0}$ множество, состоящее из восьми 7-мерных булевых векторов

$\{1111101,0000101,0111001,1000001,0001100,1110100,1001000,0110000\}$, и через $A_{1}$ множество, состоящее из восьми 7-мерных булевых векторов $\{1101110,1100111,0100011,0010110,0101010,1010010,1011011,0100011\}$.

Теорема 2. Если 7-мерный вектор $\left(a_{i}, a_{i+k}, a_{i+2 k}, a_{i+3 k}, a_{i+n}, a_{i+n+k}, a_{i+n+2 k}\right)$ принадлежит множеству $A_{0}$, то из соответствующих уравнений системь (9) следует, что

$$
X_{i+n+k}=Y_{i+n+k}=Z_{i+n+k}=0 .
$$

Если же этот вектор принадлежит множеству $A_{1}$, то

$$
X_{i+n+k}=Y_{i+n+k}=Z_{i+n+k}=1 .
$$

Доказательство теоремы основывается на двух приведённых выше примерах в леммах 1, 2 и трёх очевидных фактах:

1. Если $a_{i+k}=1, X_{i+k}+Y_{i+k}+Z_{i+k}=2$ и $a_{i+2 k}=1, X_{i+2 k}+Y_{i+2 k}+Z_{i+2 k}=2$, то $X_{i+n+k}+Y_{i+n+k}+Z_{i+n+k}=0,\left(X_{i+k}, Y_{i+k}, Z_{i+k}\right)=\left(X_{i+2 k}, Y_{i+2 k}, Z_{i+2 k}\right)$ при $a_{i+n+k}=0$.

2. Если $a_{i+k}=1, \quad X_{i+k}+Y_{i+k}+Z_{i+k}=2, a_{i+2 k}=0, X_{i+2 k}+Y_{i+2 k}+Z_{i+2 k}=1$, то $X_{i+n+k}=Y_{i+n+k}=Z_{i+n+k}=1$ при $a_{i+n+k}=1$.

3. Если $a_{i+k}=0, a_{i+2 k}=0, X_{i+k}+Y_{i+k}+Z_{i+k}=1, \quad X_{i+2 k}+Y_{i+2 k}+Z_{i+2 k}=1$, то $X_{i+n+k}=Y_{i+n+k}=Z_{i+n+k}=0$ при $a_{i+n+k}=0$.

Заметим, что повторов среди определившихся троек $\left(X_{i+n+k}, Y_{i+n+k}, Z_{i+n+k}\right)$ не будет.

Для $t>2 n$ можно рассматривать подсистему $D_{i}$, составленную из трёх уравнений с номерами $i, i+2 k, i+2 n$. Все результаты, полученные для систем $S_{j}$, естественным образом переносятся на системы $D_{i}$, в том числе и при частичной замене. 
Если система уравнений (9) является случайной и её правая часть состоит из независимых и равновероятно распределённых на элементах 0,1 случайных величин, то среднее число благоприятных комбинаций из множества $A_{0} \cup A_{1}$ выражается формулой

$$
(t-n-2 k) \cdot 16 / 2^{7}=(t-n-2 k) / 8 .
$$

Для ЗСССУ результат иной. Пусть все 12 линейных форм из уравнений с номерами $i, i+k, i+2 k, i+3 k$ линейно независимы и их значения случайные величины, равномерно распределенные на $\{0,1\}$ для любого $i \in\{1,2, \ldots, t-3 k\}$. Тогда среднее число благоприятных комбинаций из множества $A_{0} \cup A_{1}$ равно

$$
(t-n-2 k) \cdot 12 \cdot 16 / 2^{12}=3(t-n-2 k) / 64 .
$$

Приведённые факты могут быть использованы в критерии различения ССУ и ЗСССУ, так как отношение приведённых чисел равно 8/3. Другой критерий будет приведён в § 7.

Для $t>2 n$ приведём таблицу 6-мерных векторов $\bar{a}_{i}=\left(a_{i}, a_{i+k}, a_{i+2 k}, a_{i+n}, a_{i+n+k}, a_{i+2 n}\right)$, для которых $\left(X_{j}, Y_{j}, Z_{j}\right)=(0,0,0)$ при некотором $j \in\{i, i+k, i+2 k, i+n, i+n+k, i+2 n\}$. Идея доказательства такая же, как и в теореме 2.

Таблица 1

\begin{tabular}{|c|c|}
\hline$(X, Y, Z)=(0,0,0)$ & $\bar{a}_{i}=\left(a_{i}, a_{i+k}, a_{i+2 k}, a_{i+n}, a_{i+n+k}, a_{i+2 n}\right)$ \\
\hline$\left(X_{i}, Y_{i}, Z_{i}\right)$ & $B_{i}=\{(011111),(001001),(010100),(000010)\}$ \\
\hline$\left(X_{i+k}, Y_{i+k}, Z_{i+k}\right)$ & $B_{i+k}=\{(101111),(000001),(001010),(100100)\}$ \\
\hline$\left(X_{i+2 k}, Y_{i+2 k}, Z_{i+2 k}\right)$ & $B_{i+2 k}=\{(110111),(000100),(010010),(100001)\}$ \\
\hline$\left(X_{i+n}, Y_{i+n}, Z_{i+n}\right)$ & $B_{i+n}=\{(111011),(001000),(000011),(110000)\}$ \\
\hline$\left(X_{i+n+k}, Y_{i+n+k}, Z_{i+n+k}\right)$ & $B_{i+n+k}=\{(111101),(100000),(000101),(011000)\}$ \\
\hline$\left(X_{i+2 n}, Y_{i+2 n}, Z_{i+2 n}\right)$ & $B_{i+2 n}=\{(111110),(010000),(101000),(000110)\}$ \\
\hline
\end{tabular}


Аналогично строится таблица для $\left(X_{j}, Y_{j}, Z_{j}\right)=(1,1,1)$.

Таблица 2

\begin{tabular}{|c|c|}
\hline$(X, Y, Z)=(1,1,1)$ & $\bar{a}_{i}=\left(a_{i}, a_{i+k}, a_{i+2 k}, a_{i+n}, a_{i+n+k}, a_{i+2 n}\right)$ \\
\hline$\left(X_{i}, Y_{i}, Z_{i}\right)$ & $C_{i}=\{(111010),(100111),(101100),(110001)\}$ \\
\hline$\left(X_{i+k}, Y_{i+k}, Z_{i+k}\right)$ & $C_{i+k}=\{(111001),(010111),(011100),(110010)\}$ \\
\hline$\left(X_{i+2 k}, Y_{i+2 k}, Z_{i+2 k}\right)$ & $C_{i+2 k}=\{(111100),(001111),(101010),(011001)\}$ \\
\hline$\left(X_{i+n}, Y_{i+n}, Z_{i+n}\right)$ & $C_{i+n}=\{(101101),(011110),(010101),(100110)\}$ \\
\hline$\left(X_{i+n+k}, Y_{i+n+k}, Z_{i+n+k}\right)$ & $C_{i+n+k}=\{(101011),(110110),(010011),(001110)\}$ \\
\hline$\left(X_{i+2 n}, Y_{i+2 n}, Z_{i+2 n}\right)$ & $C_{i+2 n}=\{(011011),(110101),(001101),(100011)\}$ \\
\hline
\end{tabular}

Сформулируем полученный результат.

Теорема 3. Пусть $j \in\{i, i+k, i+2 k, i+n, i+n+k, i+2 n\} . \quad$ Тогдa $\left(X_{j}, Y_{j}, Z_{j}\right)=(0,0,0)$, если $\bar{a}_{i} \in B_{j}, u\left(X_{j}, Y_{j}, Z_{j}\right)=(1,1,1)$, если $\bar{a}_{i} \in C_{j}$.

К теоремам 2, 3 сделаем несколько замечаний. Если $t \leq 2 n$, то используем только теорему 2 , причём тройка $\left(X_{i+n+k}, Y_{i+n+k}, Z_{i+n+k}\right)$ может определиться только один раз. Для $t=3 n$ по теореме 3 тройки $\left(X_{i+2 n}, Y_{i+2 n}, Z_{i+2 n}\right)$ могут определиться по два раза, а остальные тройки могут встречаться и по три раза.

Среднее число благоприятных комбинаций по теореме 3 для ССУ при $t \geq 2 n+1$ равно

$$
48(t-2 n) / 2^{6}=3(t-2 n) / 4,
$$

так как в таблицах 1, 2 приведены 48 разных векторов из $2^{6}$ возможных. Если перед нами ЗСССУ, то при $t \geq(2 n+1)$ среднее число благоприятных комбинаций $N(k, n, t-2 n)$ согласно теореме 3 удовлетворяет неравенствам

$$
9(t-2 n) / 32 \leq N(k, n, t-2 n) \leq 9(t-2 n) / 16,
$$


так как из $2^{9}$ возможных значений троек нас удовлетворяют согласно таблицам 1,2 не более 6.48 значений.

На простейшем примере продемонстрируем различные приёмы определения знаков рекурренты, связанных общим уравнением

$$
x_{i} \oplus x_{i+5} x_{i+23}=a_{i}, i=1,2, \ldots, 51
$$

и рекуррентным соотношением

$$
x_{j} \oplus x_{j+7} \oplus x_{j+31}=0, j \geq 1 .
$$

Знаки $a_{1}, a_{2}, \ldots, a_{51}$ известны.

123456789012345678901234567890123456789012345678901

$x_{i} \quad 010000101101011100111100010100100101001010010010001$

$x_{i+5} \quad 010110101110011110001010010010100101001001000101010$

$x_{i+23} 001010010010100101001001000101010111101100011011100$

$a_{i} \quad 010010101111011000110100010100100000000010010011001$

Вначале, используя теорему 2, находим:

$$
\begin{array}{r}
\left(a_{3}, a_{10}, a_{17}, a_{24}, a_{34}, a_{41}, a_{48}\right)=(0100011) \in A_{1}, \\
X_{41}=x_{41}=1, Y_{41}=x_{41} \oplus x_{46}=1, Z_{41}=x_{41} \oplus x_{64}=1 .
\end{array}
$$

Это означает, что

$$
\begin{gathered}
x_{41}=1, x_{46}=0, x_{64}=x_{2} \oplus x_{16}=x_{33} \oplus x_{40}=0, \\
\varepsilon_{41}=x_{46} \cdot x_{64}=0, \varepsilon_{46}=x_{51} \cdot x_{69}=0 .
\end{gathered}
$$

Так как $x_{46}=0$, то из 23-го уравнения

$$
x_{23} \oplus x_{28} \cdot x_{46}=a_{23}=0
$$

находим $x_{23}=a_{23}=0, \varepsilon_{23}=0$. Аналогично из уравнения

$$
x_{18} \oplus x_{23} \cdot x_{41}=a_{18}=0
$$

определяем $x_{18}=0, \varepsilon_{18}=0$. Далее тем же способом находим

$$
x_{13}=0, \varepsilon_{13}=0, x_{8}=0, \varepsilon_{8}=0, x_{3}=0, \varepsilon_{3}=0 \text {. }
$$

Далее возможны следующие способы решения задачи. Первый из них связан с решением линейной системы уравнений, состоящих из двух или трёх знаков рекурренты или ошибок (произведений двух знаков рекурренты). Эти уравнения мы получаем после подстановки определившихся знаков или оши- 
бок в линейные соотношения, состоящие из трёх или четырёх членов. В частности, для нашего примера относительно ошибок известно 8 линейных трёхчленных псевдобулевых уравнений с правой частью, равной 1 , и 12 линейных трёхчленных булевых уравнений с правой частью, равной 0. Подставляя в эти 20 уравнений найденные значения ошибок $\varepsilon_{3}=0, \varepsilon_{8}=0, \varepsilon_{13}=0, \varepsilon_{18}=0, \varepsilon_{23}=0, \varepsilon_{41}=0, \varepsilon_{46}=0$, получим 4 двучленных уравнения с правой частью, равной 1 , и 4 булевых двучленных уравнения с правой частью, равной 0 . Аналогично находим 11 двучленных уравнений для знаков рекурренты. Такие системы линейных уравнений с двумя и тремя неизвестными в каждом уравнении эффективно решаются методом задания значений нескольким неизвестным [8].

Второй способ состоит в определении новых знаков рекурренты и ошибок с помощью трёхчленных и четырёхчленных линейных уравнений относительно знаков рекурренты и ошибок, а также на знании правых частей исходной системы уравнений. Дополнительно можно воспользоваться замечанием перед теоремой 2 и задать две тройки неизвестных.

Третий способ состоит в нахождении апостериорных распределений для отдельных (или некоторой совокупности) знаков рекурренты или ошибок (см. п. 2). Наиболее надёжные варианты используются для нахождения решения либо для доказательства, что это был ложный вариант задания. В нашем случае находим, что

$$
\begin{gathered}
\mathbf{P}\left(\varepsilon_{11}=1 \mid \varepsilon_{11}+\varepsilon_{18}+\varepsilon_{42}=1, \varepsilon_{4}+\varepsilon_{11}+\varepsilon_{35}=1\right) \approx 0,65, \\
\mathbf{P}\left(\varepsilon_{16}=1 \mid \varepsilon_{11}=x_{16} x_{34}=1, a_{16}=0\right)=1, \mathbf{P}\left(\varepsilon_{34}=1 \mid \varepsilon_{11}=1, a_{34}=0\right)=1, \\
\mathbf{P}\left(\varepsilon_{21}=1 \mid \varepsilon_{16}=1, a_{21}=0\right)=1, \mathbf{P}\left(\varepsilon_{26}=0 \mid \varepsilon_{21}=1, a_{26}=1\right)=1 .
\end{gathered}
$$

Если предположить, что $\varepsilon_{11}=1$, то

$$
\begin{gathered}
\varepsilon_{16}=\varepsilon_{21}=\varepsilon_{34}=1, \varepsilon_{26}=0, \varepsilon_{57}=0, \\
x_{16}=x_{34}=x_{21}=x_{39}=1, x_{26}=1, x_{44}=1,
\end{gathered}
$$

и далее, используя лишь трёхчленные соотношения для членов рекурренты и исходные нелинейные уравнения, определяем значения всех членов рекурренты.

Целесообразно для $t>3 n$ рассмотреть и более сложные конфигурации, например, составленные из подсистем $S_{i}, S_{i+k}, S_{i+2 k}, S_{i+n}, S_{i+n+k}, S_{i+2 n}$. Находим все решения и пытаемся их продолжить, если возможно, в обе стороны. С каждой стороны, если $i>k, i+3 n+k \leq t$, имеем три проверочных уравнения для каждого варианта продолжения. Так как таких вариантов для каждого решения не более трёх, то некоторые варианты продолжения могут быть отбракованы. Если число вариантов продолжения начинает возрастать, то пере- 
ходим к решению другой подсистемы. Затем пытаемся объединить решения двух ближайших подсистем.

Дополнительно следует заметить, что найденные значения линейных форм подставляем в уравнения, конфигурации, рекуррентные соотношения разной длины и упрощаем уравнения либо находим значения других линейных форм.

Таких конфигураций, как рассмотренных, так и более сложных, будет много, поэтому существует возможность определения значений линейных форм $X_{i}, Y_{i}, Z_{i}, i=1,2, \ldots, t$, без применения вычислительной техники, используя только соответствующую маркировку исходного материала. решения.

В следующем параграфе будет предложен более эффективный метод

\section{§ 4. Восстановление искажённой рекурренты}

Рассмотрим систему уравнений

$$
L_{1}\left(\bar{x}_{i}\right) \oplus L_{2}\left(\bar{y}_{i}\right) L_{3}\left(\bar{z}_{i}\right)=a_{i}, i=1,2, \ldots, t .
$$

Как уже отмечалось в § 1 (теорема 1$)$,

$$
L_{1}\left(\bar{x}_{i}\right)+\left[L_{1}\left(\bar{x}_{i}\right) \oplus L_{2}\left(\bar{y}_{i}\right)\right]+\left[L_{1}\left(\bar{x}_{i}\right) \oplus L_{3}\left(\bar{z}_{i}\right)\right] \leq 1
$$

при $a_{i}=0$ и

$$
L_{1}\left(\bar{x}_{i}\right)+\left[L_{1}\left(\bar{x}_{i}\right) \oplus L_{2}\left(\bar{y}_{i}\right)\right]+\left[L_{1}\left(\bar{x}_{i}\right) \oplus L_{3}\left(\bar{z}_{i}\right)\right] \geq 2,
$$

если $a_{i}=1$. Поэтому некоторые результаты из $\S 3$ переносятся и на этот случай, так как

$$
X_{i}=L_{1}\left(\bar{x}_{i}\right), \quad Y_{i}=L_{1}\left(\bar{x}_{i}\right) \oplus L_{2}\left(\bar{y}_{i}\right), \quad Z_{i}=L_{1}\left(\bar{x}_{i}\right) \oplus L_{3}\left(\bar{z}_{i}\right) .
$$

Например, если мы нашли, что

$$
X_{i+2 n}=Y_{i+2 n}=Z_{i+2 n}=0,
$$

то это означает для нашего случая справедливость равенств

$$
L_{1}\left(\bar{x}_{i+2 n}\right)=L_{2}\left(\bar{y}_{i+2 n}\right)=L_{3}\left(\bar{z}_{i+2 n}\right)=0 .
$$

Если же

$$
X_{i+2 n}=Y_{i+2 n}=Z_{i+2 n}=1 \text {, }
$$

To

$$
L_{1}\left(\bar{x}_{i+2 n}\right)=1, L_{2}\left(\bar{y}_{i+2 n}\right)=0, L_{3}\left(\bar{z}_{i+2 n}\right)=0 .
$$


Ниже будет изложен иной подход к решению таких систем уравнений. По существу мы будем изучать систему линейных уравнений с ошибками в правой части. Причём эти ошибки удовлетворяют некоторым линейным булевым и псевдобулевым уравнениям.

Введём, как и в $~ 2$, обозначения

$$
L_{2}\left(\bar{y}_{i}\right) \cdot L_{3}\left(\bar{z}_{i}\right)=\varepsilon_{i}, \quad i=1,2, \ldots, t .
$$

Если все три последовательности $\bar{X}, \bar{Y}, \bar{Z}$ удовлетворяют трёхчленному равенству (6), то

$$
\varepsilon_{i} \oplus \varepsilon_{i+k} \oplus \varepsilon_{i+n}=a_{i} \oplus a_{i+k} \oplus a_{i+n}
$$

и верны следующие утверждения.

Пусть $t=2 n$. Найдём все четырёхчленные соотношения для $2 n$ членов рекурренты (6), которые являются суммами двух трехчленных соотношений вида

$$
x_{i} \oplus x_{i+k} \oplus x_{i+n}=0 .
$$

Таких четырёхчленов будет $n$ :

$$
\begin{gathered}
x_{i} \oplus x_{i+2 k} \oplus x_{i+n} \oplus x_{i+k+n}=0,1 \leq i \leq n-k, \\
x_{i} \oplus x_{i+k} \oplus x_{i+n-k} \oplus x_{i+2 n-k}=0,1 \leq i \leq k .
\end{gathered}
$$

Каждый знак $x_{j}, 1 \leq j \leq 2 n$, встречается в двух четырёхчленах. Здесь уместно заметить, что в трёхчленных соотношениях знаки $x_{1}, \ldots, x_{k}$ и $x_{n+k+1}, \ldots, x_{n}$ встречаются по одному разу, а знаки $x_{k+1}, \ldots, x_{n+k}$ по два раза.

Подставим в эти $n$ четырёхчленов $x_{j}=\varepsilon_{j} \oplus \alpha_{j}$. Получим $n_{0}\left(n_{1}\right)$ булевых уравнений относительно ошибок с правой частью, равной 0 (соответственно 1).

Лемма 3. Если члены исходной трёхчленной рекурренты (6) удовлетворяют некоторым $2 s$ - или $(2 s-1)$-членным соотночениям, то число искажений на этих $2 s$ или $2 s-1$ местах не может быть равным $2 s-1$.

Замечание 3. В условиях леммы 3 булево равенство $\varepsilon_{i} \oplus \varepsilon_{i+k} \oplus \varepsilon_{i+n}=1$ сводится к псевдобулеву равенству $\varepsilon_{i}+\varepsilon_{i+k}+\varepsilon_{i+n}=1$, а булево равенство $\varepsilon_{i} \oplus \varepsilon_{i+2 k} \oplus \varepsilon_{i+n} \oplus \varepsilon_{i+n+k}=1$ сводится к псевдобулеву равенству $\varepsilon_{i}+\varepsilon_{i+2 k}+$ $+\varepsilon_{i+n}+\varepsilon_{i+n+k}=1$.

Приведём примеры использования этих утверждений. Пусть

$$
\begin{gathered}
\varepsilon_{i} \oplus \varepsilon_{i+2 k} \oplus \varepsilon_{i+2 n}=0, \\
\varepsilon_{i+n} \oplus \varepsilon_{i+n+k} \oplus \varepsilon_{i+2 n}=1 .
\end{gathered}
$$


Тогда выполняются два псевдобулевых равенства

$$
\begin{gathered}
\varepsilon_{i+n}+\varepsilon_{i+n+k}+\varepsilon_{i+2 n}=1, \\
\varepsilon_{i}+\varepsilon_{i+k}+\varepsilon_{i+n}+\varepsilon_{i+n+k}=1
\end{gathered}
$$

Лемма 4. Система из трёх булевых линейных уравнений с семью неизвестными

$$
\begin{gathered}
\varepsilon_{i} \oplus \varepsilon_{i+k} \oplus \varepsilon_{i+n}=0, \\
\varepsilon_{i+k} \oplus \varepsilon_{i+2 k} \oplus \varepsilon_{i+n+k}=1, \\
\varepsilon_{i+2 k} \oplus \varepsilon_{i+3 k} \oplus \varepsilon_{i+n+2 k}=0,
\end{gathered}
$$

имеющая 16 решений, как система уравнений относительно ошибок заменяется системой из трёх псевдобулевых линейных уравнений

$$
\begin{gathered}
\varepsilon_{i}+\varepsilon_{i+2 k}+\varepsilon_{i+n}+\varepsilon_{i+n+k}=1, \\
\varepsilon_{i+k}+\varepsilon_{i+2 k}+\varepsilon_{i+n+k}=1, \\
\varepsilon_{i+k}+\varepsilon_{i+3 k}+\varepsilon_{i+n+k}+\varepsilon_{i+n+2 k}=1,
\end{gathered}
$$

имеющей пять решений относительно семи неизвестных.

Доказательство. Булева система (13) заменяется эквивалентной булевой системой

$$
\begin{gathered}
\varepsilon_{i} \oplus \varepsilon_{i+2 k} \oplus \varepsilon_{i+n} \oplus \varepsilon_{i+n+k}=1, \\
\varepsilon_{i+k} \oplus \varepsilon_{i+2 k} \oplus \varepsilon_{i+n+k}=1, \\
\varepsilon_{i+k} \oplus \varepsilon_{i+3 k} \oplus \varepsilon_{i+n+k} \oplus \varepsilon_{i+n+2 k}=1 .
\end{gathered}
$$

Эта система согласно утверждению леммы 3 и замечанию 3 сводится к псевдобулевой системе (14), имеющей пять решений относительно семи неизвестных.

Аналогично рассматривается система (13) с другой правой частью.

Лемма 5. Система из трёх булевых линейных уравнений

$$
\begin{gathered}
\varepsilon_{i} \oplus \varepsilon_{i+k} \oplus \varepsilon_{i+n}=1, \\
\varepsilon_{i+k} \oplus \varepsilon_{i+2 k} \oplus \varepsilon_{i+n+k}=0, \\
\varepsilon_{i+2 k} \oplus \varepsilon_{i+3 k} \oplus \varepsilon_{i+n+2 k}=1
\end{gathered}
$$

заменяется (как система уравнений относительно ошибок) системой из четырёх псевдобулевых линейных уравнений

$$
\varepsilon_{i}+\varepsilon_{i+k}+\varepsilon_{i+n}=1
$$




$$
\begin{gathered}
\varepsilon_{i}+\varepsilon_{i+2 k}+\varepsilon_{i+n}+\varepsilon_{i+n+k}=1, \\
\varepsilon_{i+n}+\varepsilon_{i+3 k}+\varepsilon_{i+n+k}+\varepsilon_{i+n+2 k}=1, \\
\varepsilon_{i+2 k}+\varepsilon_{i+3 k}+\varepsilon_{i+n+2 k}=1,
\end{gathered}
$$

имеющей пять решений относительно семи неизвестных, причём во всех решениях $\varepsilon_{i+n+k}=0$.

Доказательство. Вначале, как и в лемме 4, булева система заменяется псевдобулевой, находятся пять решений. Если $\varepsilon_{i+n+k}=1$, то все остальные шесть ошибок равны 0 и поэтому не удовлетворяются первое и последнее уравнения. По существу это утверждение является улучшением теоремы 2.

Замечание 4. Равенство $\varepsilon_{i+n+k}=0$ можно доказать методом выделения и оценки отдельных неизвестных [5]. Для этого достаточно сложить второе и третье уравнения и вычесть из этой суммы первое и четвёртое уравнения. Получим $2 \varepsilon_{i+n+k}=0$.

Для $t>2 n$ можно воспользоваться следующим утверждением.

Лемма 6. Система из трёх линейных булевых уравнений с шестью неизвестныли

$$
\begin{gathered}
\varepsilon_{i} \oplus \varepsilon_{i+k} \oplus \varepsilon_{i+n}=c_{i}, \\
\varepsilon_{i+k} \oplus \varepsilon_{i+2 k} \oplus \varepsilon_{i+n+k}=c_{i+k}, \\
\varepsilon_{i+n} \oplus \varepsilon_{i+n+k} \oplus \varepsilon_{i+2 n}=c_{i+n}
\end{gathered}
$$

как система уравнений относительно ошибок имеет четыре решения для $c_{i}=c_{i+k}=c_{i+n}=1,3$ решения при $c_{i}+c_{i+k}+c_{i+n} \in\{1,2\}$ и восемь решений для $c_{i}=c_{i+k}=c_{i+n}=0$.

Доказательство. Согласно леммам 3 и 4 в первом случае мы рассматриваем псевдобулеву систему, имеющую четыре решения. Во втором случае булева система из трёх трёхчленных уравнений заменяется псевдобулевой системой с правыми частями, равными единицам. Во втором случае будет 3 решения, в третьем случае булева система не меняется и поэтому имеет восемь решений.

Таким образом, используя теоремы 2,3 и леммы $3,4,5,6$, мы сводим задачу нахождения решений нелинейной системы (12) к задаче решения системы из линейных псевдобулевых уравнений, содержащих две, три или четыре ошибки, и имеющей меньшее число неизвестных.

\section{§ 5. Четырёхчленная рекуррента}

В этом случае методы, изложенные в $§ 3$ (теоремы 2 и 3), не работают. Поэтому приходится на первом этапе задавать некоторое число неизвестных или ошибок. 
Рассмотрим пример. Пусть

$$
x_{i} \oplus x_{i+10} \oplus x_{i+19} \oplus x_{i+31}=0,
$$

а система уравнений

$$
x_{i} \oplus x_{i+7} \cdot x_{i+13}=a_{i}, i=1,2, \ldots, 51 .
$$

123456789012345678901234567890123456789012345678901

$$
\begin{array}{ll}
x_{i} & 111000011101001100101111001110011000100101000101000 \\
x_{i+7} & 111010011001011110011100110101001010001010000011101 \\
x_{i+13} & 011001011110011100110101001010001010000011101101001 \\
a_{i} & 100000000101010000111011001110010000100111000100001
\end{array}
$$

Приведём восемь четырёхчленных соотношений между ошибками, правые части у которых равны 1:

$$
\begin{aligned}
& \varepsilon_{1}+\varepsilon_{11}+\varepsilon_{20}+\varepsilon_{32}=1, \\
& \varepsilon_{5}+\varepsilon_{15}+\varepsilon_{24}+\varepsilon_{36}=1, \\
& \varepsilon_{6}+\varepsilon_{16}+\varepsilon_{25}+\varepsilon_{37}=1, \\
& \varepsilon_{8}+\varepsilon_{18}+\varepsilon_{27}+\varepsilon_{39}=1, \\
& \varepsilon_{9}+\varepsilon_{19}+\varepsilon_{28}+\varepsilon_{40}=1, \\
& \varepsilon_{12}+\varepsilon_{22}+\varepsilon_{31}+\varepsilon_{43}=1, \\
& \varepsilon_{15}+\varepsilon_{25}+\varepsilon_{34}+\varepsilon_{46}=1, \\
& \varepsilon_{17}+\varepsilon_{27}+\varepsilon_{36}+\varepsilon_{48}=1
\end{aligned}
$$

В эти уравнения ошибки $\varepsilon_{15}, \varepsilon_{25}, \varepsilon_{27}, \varepsilon_{36}$ входят по два раза.

Положим $\varepsilon_{15}=1$. Тогда находим $\varepsilon_{5}=\varepsilon_{24}=\varepsilon_{25}=\varepsilon_{34}=\varepsilon_{36}=\varepsilon_{46}=0$ и соответственно из уравнений (15) определяем $x_{5}=0, x_{15}=1, x_{24}=1$, $x_{25}=0, x_{34}=0, x_{36}=0, x_{46}=1$. Подставим эти значения в нелинейную часть уравнений (15). Дополнительно находим

$$
\begin{gathered}
x_{2} \oplus x_{9}=0, x_{8} \oplus x_{21}=0, x_{11} \oplus x_{18}=0, x_{17} \oplus x_{30}=0, \\
x_{33} \oplus x_{40}=0, x_{39} \oplus x_{52}=0, \\
x_{12}=1, x_{18}=0, x_{21}=1, x_{23}=1, x_{27}=1, x_{29}=1, x_{11}=0, x_{8}=1 .
\end{gathered}
$$


Найденные значения пятнадцати членов рекурренты подставляем в исходные уравнения и находим значения всех членов рекурренты. В случае, когда решения нет, полагаем $\varepsilon_{15}=0$ и, например $x_{25}=1$.

Если в нелинейном члене уравнения (15) вместо одного члена рекурренты находится известная линейная форма от членов рекурренты, то задача вначале решается аналогичным образом, но, возможно, придётся решать линейную систему уравнений от сравнительно небольшого числа неизвестных.

Самый сложный случай - это когда в уравнении (15) вместо нелинейного члена стоит произведение двух известных линейных форм. В этом случае целесообразно вначале решить несколько уравнений относительно ошибок. В частности, число решений подсистемы относительно 10 неизвестных, образованной 2-м, 7-м, 8-м уравнениями из приведённых восьми, равно 24.

\section{§ 6. Критерий различения случайной равновероятной последовательности и искажённой трёхчленной рекурренты}

Пусть гипотеза $H_{0}$ состоит в том, что последовательность $a_{1}, a_{2}, \ldots, a_{t}$ порождается системой уравнений (12), а гипотеза $H_{1}-$ в том, что члены этой последовательности случайны, независимы и равновероятны. Как уже отмечалось в § 3 ,

$$
\mathbf{P}\left(\varepsilon_{i} \oplus \varepsilon_{i+k} \oplus \varepsilon_{i+n}=a_{i} \oplus a_{i+k} \oplus a_{i+n}=0 \mid H_{0}\right)=\frac{5}{8}, \quad i=1,2, \ldots, t-n .
$$

Для гипотезы $H_{1}$ эта вероятность равна $1 / 2$.

Обозначим через $\eta$ число событий $\left\{a_{i} \oplus a_{i+k} \oplus a_{i+n}=0\right\}$. Очевидно, что

$$
\mathbf{M}_{0} \eta=5(t-n) / 8, \quad \mathbf{M}_{1} \eta=(t-n) / 2, \quad \mathbf{D}_{1} \eta=(t-n) / 4 .
$$

Следовательно, нормированное расстояние между средними значениями $\eta$ при этих гипотезах равно

$$
\rho\left(H_{0}, H_{1}\right)=\left(\mathbf{M}_{0} \eta-\mathbf{M}_{1} \eta\right) / \sqrt{\mathbf{D}_{1} \eta}=\frac{1}{4} \sqrt{(t-n)} .
$$

Если $t=2 n$, то $\rho\left(H_{0}, H_{1}\right) \geq 3$ при $n \geq 144$.

Исходные последовательности для двух примеров были взяты из таблицы случайных чисел, приведённой в учебнике В. П. Чистякова [9].

Автор благодарен Ф. М. Малышеву, А. М. Зубкову, В. Г. Смирнову за ряд замечаний. 


\section{Список литературы}

1. Балакин Г. В. Системы уравнений с мешающими параметрами // Труды по дискретной математике. - Т. 10. - М.: ФИЗМАТЛИТ, 2007. - С. 7 17.

2. Балакин Г. В. Системы булевых уравнений с искаженной правой частью при ограничениях на значения неизвестных и ошибок // Труды по дискретной математике. - Т. 11. Вып. 1. - М.: ФИЗМАТЛИТ, 2008. C. $5-17$.

3. Балакин Г. В. Случайные системы уравнений и их криптографические приложения // Математика и безопасность информационных технологий: Материалы конф. в МГУ 23-24 октября 2003 г. - М.: МЦНМО, 2004. - C. 53-73.

4. Балакин Г. В. Линейные псевдобулевые неравенства // Математические вопросы криптографии. - 2010. - Т. 1. Вып. 3. - С. 5-18.

5. Балакин $Г$. В. О возможности решения систем линейных целочисленных уравнений методом выделения и оценки отдельных неизвестных // Дискретная математика. - 1994. - Т. 6. Вып. 1. - С. 116-126.

6. Балакин Г. В. О вероятностном подходе к решению систем уравнений с целочисленными неизвестными // Дискретная математика. - 1995. T. 7. Вып. 1. - С. 88-98.

7. Смирнов В. Г. Системы булевых уравнений рекуррентного типа // Обозрение пром. и прикл. матем. - 1995. - Т. 2. Вып. 3. - С. 477-482.

8. Балакин Г. В., Бачурин, С. А. Оценка параметров последовательного подбора неизвестных // Труды по дискретной математике. - Т. 6. - М.: ФИЗМАТЛИТ, 2002. - С. 7-13.

9. Чистяков В. П. Курс теории вероятностей. - 5-е изд. - М.: Агар, 2000. $-256 \mathrm{c}$. 